\title{
Unilateral pallidothalamic tractotomy for akinetic-rigid Parkinson's disease: a prospective open-label study
}

\author{
Shiro Horisawa, MD, ${ }^{1}$ Atsushi Fukui, MD, PhD, ${ }^{1}$ Hayato Yamahata, MD, ${ }^{1}$ Yukiko Tanaka, MD,1 \\ Atsushi Kuwano, MD, ${ }^{1}$ Oji Momosaki, MD, ${ }^{1}$ Mutsumi lijima, MD, PhD, ${ }^{2}$ Magi Nanke, MD, ${ }^{3}$ \\ Takakazu Kawamata, MD, PhD, ${ }^{1}$ and Takaomi Taira, MD, PhD'
}

Departments of ${ }^{1}$ Neurosurgery and ${ }^{2}$ Neurology, and ${ }^{3}$ School of Medicine, Tokyo Women's Medical University, Tokyo, Japan

\begin{abstract}
OBJECTIVE Neurosurgical ablation is an effective treatment for medically refractory motor symptoms of Parkinson's disease (PD). A limited number of studies have reported the effect of ablation of the pallidothalamic tract for PD. In this study, the authors evaluated the safety and efficacy of unilateral pallidothalamic tractotomy for akinetic-rigid (AR)-PD.

METHODS Fourteen AR-PD patients, who were enrolled in this prospective open-label study, underwent unilateral pallidothalamic tractotomy. The Movement Disorder Society-Unified Parkinson's Disease Rating Scale (MDS-UPDRS) Part III and Part IV (dyskinesia and dystonia) scores and levodopa equivalent daily dose (LEDD) were evaluated at baseline and at 3 and 12 months postoperatively.
\end{abstract}

RESULTS Of the 14 patients enrolled in the study, 4 were lost to follow-up and 10 were analyzed. The total MDSUPDRS Part III score significantly improved from $45 \pm 4.6$ at baseline to $32.9 \pm 4.8$ at 12 months postoperatively ( $p=$ 0.005 ). Contralateral side rigidity and bradykinesia significantly improved from $4.4 \pm 0.5$ and $10.4 \pm 1.5$ at baseline to 1.7 $\pm 0.4(p=0.005)$ and $5.2 \pm 1.4(p=0.011)$ at 12 months, respectively. While posture significantly improved with a $20 \%$ reduction in scores $(p=0.038)$, no significant improvement was found in gait $(p=0.066)$. Dyskinesia and dystonia were improved with a $79.2 \%(p=0.0012)$ and $91.7 \%(p=0.041)$ reduction in scores, respectively. No significant change was found in the LEDD. Hypophonia was noted in 2 patients, eyelid apraxia was noted in 1 patient, and a reduced response to levodopa, which resulted in an increase in the daily dose of levodopa, was noted in 3 patients. No serious permanent neurological deficits were observed.

CONCLUSIONS Unilateral pallidothalamic tractotomy improved contralateral side rigidity and bradykinesia, dyskinesia, and dystonia in patients with AR-PD.

Clinical trial registration no.: UMIN000031138 (umin.ac.jp)

https://thejns.org/doi/abs/10.3171/2020.7.JNS201547

KEYWORDS Parkinson's disease; pallidothalamic tract; campotomy; stereotactic neurosurgery; functional neurosurgery

$\mathrm{N}$ EUROSURGICAL ablation and deep brain stimulation (DBS) are the current standard treatments for the cardinal motor symptoms in Parkinson's disease (PD). ${ }^{1-5}$ Although the mainstay surgical treatment is DBS, ablative procedures are still an important option for patients who cannot access DBS because of economic or geographic limitations. Additionally, increased attention has been paid to recent advancements in the field of minimally invasive ablative procedures, including MRI-guided focused ultrasound ${ }^{6-8}$
The pallidothalamic tract consists of two distinct fiber bundles including the ansa lenticularis and the lenticular fasciculus, which connect the internal segment of the globus pallidus (GPi) to thalamic subnuclei such as the ventral anterior nucleus and the ventrolateral nucleus. ${ }^{9,10}$ Both fiber bundles originate from different parts of the $\mathrm{GPi}$, course across the internal capsule, and merge at the field of Forel where they form one fiber, which is called the "thalamic fasciculus."11 Ablation of the field of Forel, also called "campotomy," was first performed for the treatment

ABBREVIATIONS AC-PC = anterior commissure-posterior commissure; $\mathrm{AR}=$ akinetic-rigid; $\mathrm{DBS}=$ deep brain stimulation; $\mathrm{GPi}=$ internal segment of the globus pallidus; LEDD = levodopa equivalent daily dose; MDS-UPDRS = Movement Disorder Society-Unified Parkinson's Disease Rating Scale; MX = mixed; PD = Parkinson's disease; $\mathrm{PTT}=$ pallidothalamic tractotomy; $\mathrm{STN}=$ subthalamic nucleus; $\mathrm{TD}=$ tremor dominant.

SUBMITTED April 29, 2020. ACCEPTED July 21, 2020.

INCLUDE WHEN CITING Published online January 15, 2021; DOI: 10.3171/2020.7.JNS201547. 
of PD in the 1960s by Spiegel et al. and was later revived by Jeanmonod et al., who renamed the procedure "pallidothalamic tractotomy" (PTT). ${ }^{12,13}$ Jeanmonod's group detailed the effect of PTT with the use of radiofrequency and focused ultrasound ablation on the cardinal motor symptoms of PD. ${ }^{6,12,14}$ However, only two research groups have published reports investigating PTT/campotomy for PD. ${ }^{6,12,15}$ Additionally, these studies mainly evaluated PD with tremor. Akinetic-rigid (AR)-PD without tremor is the subtype of PD with the poorest prognosis, and the efficacy of PTT/campotomy for AR-PD has not been evaluated. To that end, in the present study, we report the prospective efficacy and safety of unilateral PTT for AR-PD.

\section{Methods \\ Study Design}

This was a prospective, open-label noncontrolled study performed at Tokyo Women's Medical University Hospital. The protocol was reviewed and approved by the Ethics Committees of Tokyo Women's Medical University Hospital. All patients provided written informed consent before inclusion in this study. The study is registered in the University Hospital Medical Information Network Clinical Trials Registry (umin.ac.jp), and its clinical trial registry number is UMIN000031138.

\section{Patients}

Patients with medically refractory PD who declined to undergo DBS surgery were enrolled. The diagnosis of PD was confirmed by a neurologist or neurosurgeon specializing in movement disorders (T.T., M.I., S.H.). The exclusion criteria were as follows: comorbid neurodegenerative disorders, history of intracranial surgery, cerebral stroke, traumatic brain injury, and antiplatelet or anticoagulant medications.

\section{Evaluations}

The primary endpoint was the change in Movement Disorder Society-Unified Parkinson's Disease Rating Scale (MDS-UPDRS) scores after unilateral PTT. The MDS-UPDRS Part III (off-medication state) and Part IV, specifically dyskinesia items 4.1-4.2 and dystonia item 4.6, were assessed. Evaluations were performed at baseline and at 3 and 12 months postoperatively. To interpret surgical outcomes clinically, subscores of the MDSUPDRS were also evaluated independently as follows: contralateral side (surgical side) and ipsilateral side rigidity (item 3.3) and bradykinesia (items 3.4-3.8), gait (items 3.10-3.11), and posture (items 3.12-3.13). The levodopa equivalent daily dose (LEDD) was also evaluated. Antiparkinsonian drugs were not changed before the surgery. After surgery, an adjustment of antiparkinsonian drugs was allowed based on the patient's neurological condition. Postoperative MRI was performed on the day of surgery and 3 months postoperatively.

\section{Surgical Procedure}

Leksell SurgiPlan (Elekta) was used to plan the stereotactic targets and electrode trajectory. Unilateral PTT was performed on the side most affected by parkinsonian motor symptoms. With the patient under local anesthesia, a Leksell stereotactic frame (Elekta) was fixed to their head. Axial (1-mm slice) T1-weighted and axial (1-mm slice) and coronal (2-mm slice) T2-weighted MR images were used to plan the PTT surgery. The lateral part of the pallidothalamic tract is long and corresponds to the thalamic fasciculus on Morel's atlas. According to Morel's atlas, the width of the pallidothalamic tract is $6 \mathrm{~mm}$ at the anterior commissure-posterior commissure (AC-PC) plane. Therefore, we set two stereotactic ablative targets to cover the pallidothalamic tract (Fig. 1). The medial target was set at $8-10 \mathrm{~mm}$ lateral and $0.5-2.5 \mathrm{~mm}$ inferior to the midpoint of the AC-PC and at the midpoint of the AC-PC. The lateral target was set at $10-13 \mathrm{~mm}$ lateral, $1.0-2.0 \mathrm{~mm}$ inferior, and $1 \mathrm{~mm}$ posterior to the midpoint of the ACPC. A monopolar radiofrequency electrode $(1.0-\mathrm{mm}$-diameter tip with an uninsulated length of 2.0-4.0 mm) and a Leksell Neuro Generator (Elekta) were used to perform macrostimulation $(130 \mathrm{~Hz}, 100-\mu \mathrm{sec}$ pulse width, up to 5 $\mathrm{mA}$ ) and ablation. Before ablation, macrostimulation was performed to confirm adverse effects. A single ablation at $70^{\circ} \mathrm{C}$ for 40 seconds was made at each target.

\section{Statistical Analysis}

A Shapiro-Wilk normality test was performed to confirm the normality of the data. As the data were nonnormally distributed, Friedman's tests were used to determine the change in MDS-UPDRS scores and the LEDD across the three study visits: at baseline and at 3 and 12 months postoperatively. Wilcoxon's signed-rank tests were also used to compare the evaluated variables between baseline and 12 months. Statistical analysis was performed using the JMP statistical package, version 13.0.0 (SAS Institute Inc.). All statistical tests were two-tailed, and a $\mathrm{p}$ value < 0.05 was determined to be statistically significant. Values are expressed as the mean \pm standard deviation, unless indicated otherwise.

\section{Results}

\section{Patients}

From December 2016 to April 2019, 14 patients with PD were enrolled in this study. Of these, 4 were lost to follow-up; therefore, 10 patients ( 5 male, 5 female) were included in the final analysis. All the patients had ARPD. The mean age at disease onset was $52.1 \pm 7.1$ years. The mean disease duration was $12.6 \pm 5.0$ years. The mean preoperative Hoehn and Yahr stage was $3.2 \pm 0.6$. Detailed information regarding patient characteristics is summarized in Table 1.

\section{Clinical Assessments}

A summary of the clinical assessments is given in Table 2 and Fig. 2. The total MDS-UPDRS Part III score was significantly improved from $45.0 \pm 4.6$ at baseline to $32.9 \pm 4.8$ at 12 months postoperatively ( $\mathrm{p}=0.005 ; 26.9 \%$ reduction). Contralateral side rigidity and bradykinesia were significantly improved from $4.4 \pm 0.5$ and $10.4 \pm 1.5$ at baseline to $1.7 \pm 0.4(p=0.005)$ and $5.2 \pm 1.4(p=0.011)$ at 12 months, respectively (Video 1 ). 

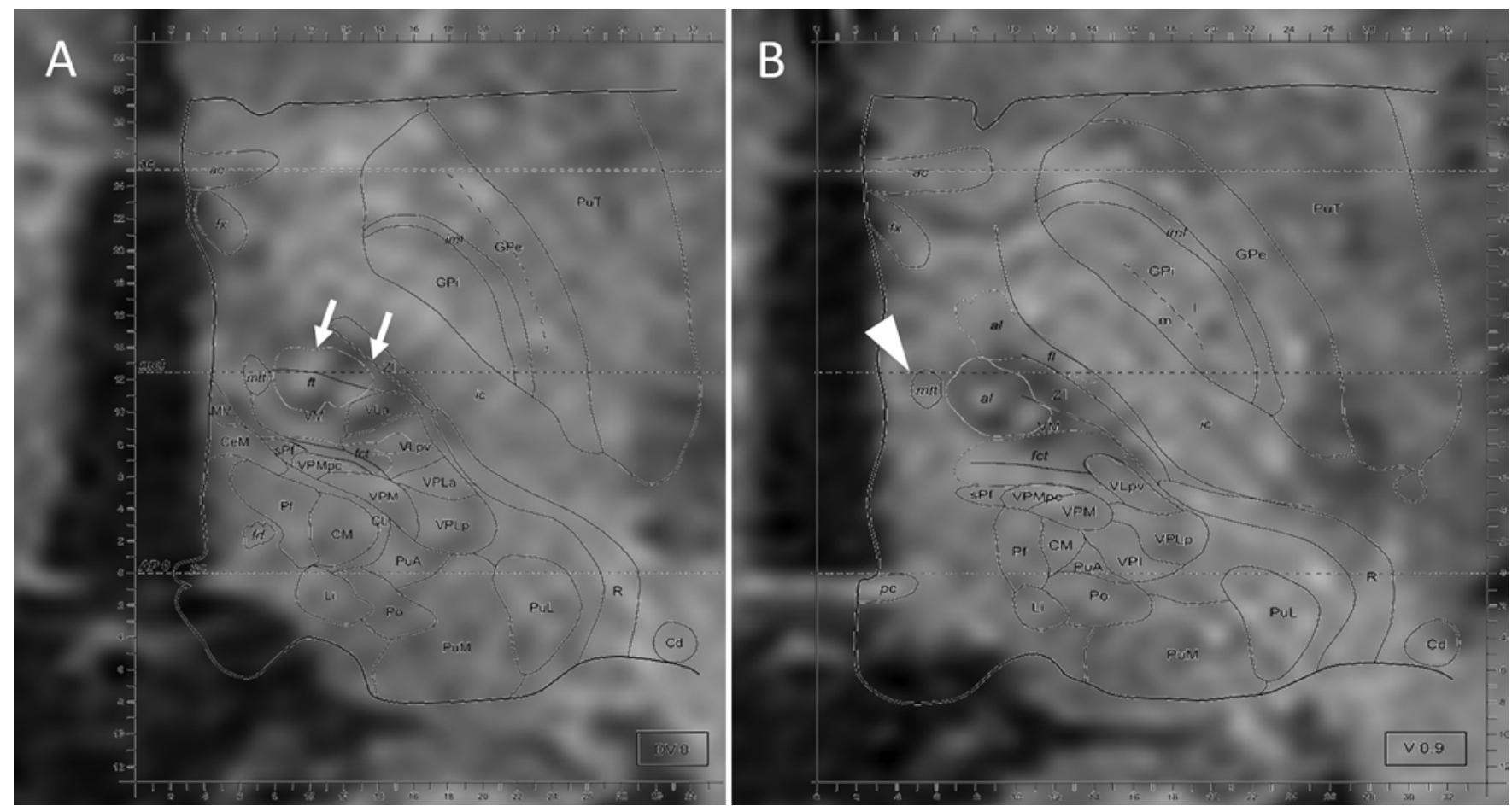

FIG. 1. Anatomical location of the pallidothalamic tract shown by postoperative MRI overlaying Morel's atlas. A: Two lesions (arrows) on the thalamic fasciculus (ft), which correspond to the pallidothalamic tract at the AC-PC plane. B: The same lesions at 0.9 $\mathrm{mm}$ below the AC-PC plane. The lesions were set just lateral to the mammillothalamic tract ( $\mathrm{mtt}$; arrowhead). al = ansa lenticularis; $\mathrm{fl}=$ lenticular fasciculus.

VIDEO 1. Pre- and postoperative bradykinesia. Copyright Shiro Horisawa. Published with permission. Click here to view.

No significant change was found in ipsilateral side rigidity and bradykinesia. Both gait and posture were significantly improved as compared among the three time points (Friedman test), but only posture was improved in a comparison between baseline and 12 months postoperatively (Video 2).

VIDEO 2. Pre- and postoperative gait in the medication-off period. Copyright Shiro Horisawa. Published with permission. Click here to view.

Dyskinesia and dystonia were significantly improved

TABLE 1. Summary of characteristics of 10 patients with AR-PD

\begin{tabular}{lc}
\hline \multicolumn{1}{c}{ Variable } & Value \\
\hline No. of patients & \\
\hline Men & 5 \\
\hline Women & $52.1 \pm 7.1$ \\
\hline Age at disease onset in yrs & $12.6 \pm 5.0$ \\
\hline Disease duration in yrs & $3.2 \pm 0.6$ \\
\hline Preop Hoehn \& Yahr stage & \\
\hline Side of surgery, $n$ & 6 \\
\hline Rt & 4 \\
\hline Lt
\end{tabular}

Values are expressed as the mean \pm standard deviation, unless indicated otherwise. with a $79.2 \%(p=0.0012 ;$ Video 3$)$ and a $91.7 \%(p=0.041)$ reduction in scores at 12 months.

VIDEO 3. Pre- and postoperative dyskinesia. Copyright Shiro

Horisawa. Published with permission. Click here to view.

The LEDD was $575 \pm 79.7 \mathrm{mg}$ at baseline and $575 \pm 92.2$ $\mathrm{mg}$ at 12 months, which was not significantly different. Postoperative MRI showed coagulated lesions located above the subthalamic nucleus (STN) in all patients, as shown in Fig. 3.

\section{Adverse Events}

All adverse events are listed in Table 3. Six patients developed somnolence during the surgery, which spontaneously improved the following morning. A reduced response to the oral intake of levodopa, including a shorter effect duration and a longer time to become effective, developed within 1 week after the surgery in 3 patients. The extended off duration resulted in an increase of their daily dose and dose frequency of levodopa. Two patients developed hypophonia, which was mild and did not interfere with their daily communication. A chronic subdural hematoma developed in 1 patient at 2 months postsurgery. The hematoma caused mild hemiparesis, and hematoma irrigation surgery was performed, which resulted in complete recovery of neurological deficits.

\section{Patients Lost to Follow-Up}

Four patients were lost to follow-up; 2 lived in remote locations, and the reason for the loss of the other 2 pa- 
TABLE 2. Clinical outcomes of 10 patients who underwent PTT for AR-PD

\begin{tabular}{|c|c|c|c|c|c|c|}
\hline MDS-UPDRS & Baseline & $3 \mathrm{Mos}$ & p Value* & 12 Mos & p Value† & p Valuef \\
\hline \multicolumn{7}{|l|}{ Part III } \\
\hline Total score & $45 \pm 4.6$ & $31.5 \pm 4.6$ & 0.005 & $32.9 \pm 4.8$ & 0.005 & $<0.001$ \\
\hline \multicolumn{7}{|l|}{ Contralat side } \\
\hline Rigidity (item 3.3) & $4.4 \pm 0.5$ & $1.7 \pm 0.4$ & 0.005 & $1.7 \pm 0.4$ & 0.005 & $<0.001$ \\
\hline Bradykinesia (items 3.4-3.8) & $10.4 \pm 1.5$ & $5.4 \pm 1.3$ & 0.008 & $5.2 \pm 1.4$ & 0.011 & $<0.001$ \\
\hline \multicolumn{7}{|l|}{ Ipsilat side } \\
\hline Rigidity (item 3.3) & $3.4 \pm 0.6$ & $3 \pm 0.6$ & 0.194 & $3 \pm 0.6$ & 0.194 & 0.368 \\
\hline Bradykinesia (items 3.4-3.8) & $7.6 \pm 1.3$ & $7.3 \pm 1.3$ & 0.180 & $7.3 \pm 1.3$ & 0.180 & 0.135 \\
\hline Gait (items 3.10-3.11) & $4.6 \pm 0.5$ & $3 \pm 0.6$ & 0.016 & $3.7 \pm 0.6$ & 0.066 & 0.004 \\
\hline Posture (items 3.12-3.13) & $4 \pm 0.4$ & $2.9 \pm 0.5$ & 0.020 & $3.2 \pm 0.5$ & 0.038 & 0.006 \\
\hline \multicolumn{7}{|l|}{ Part IV } \\
\hline Dyskinesia (items 4.1-4.2) & $4.8 \pm 0.4$ & $0.8 \pm 0.3$ & 0.011 & $1 \pm 0.3$ & 0.012 & $<0.001$ \\
\hline Dystonia (item 4.6) & $2.4 \pm 0.4$ & $0.2 \pm 0.2$ & 0.041 & $0.2 \pm 0.2$ & 0.041 & 0.007 \\
\hline LEDD (mg) & $575 \pm 79.7$ & $577.8 \pm 106$ & 0.893 & $575 \pm 92.2$ & 0.99 & 0.651 \\
\hline Hoehn \& Yahr stage & $3.2 \pm 0.2$ & & & $3 \pm 0.3$ & & \\
\hline
\end{tabular}

Data are presented as mean \pm standard deviation, unless indicated otherwise. Significance was accepted at $p<0.05$.

Boldface type indicates statistical significance. Bonferroni correction was used for multiple comparisons.

${ }^{*}$ Comparison of baseline and 3-month postoperative scores, Wilcoxon signed-rank test.

† Comparison of baseline and 12-month postoperative scores, Wilcoxon signed-rank test.

$\ddagger$ Comparison of baseline, 3-month postoperative, and 12-month postoperative scores, Friedman test.
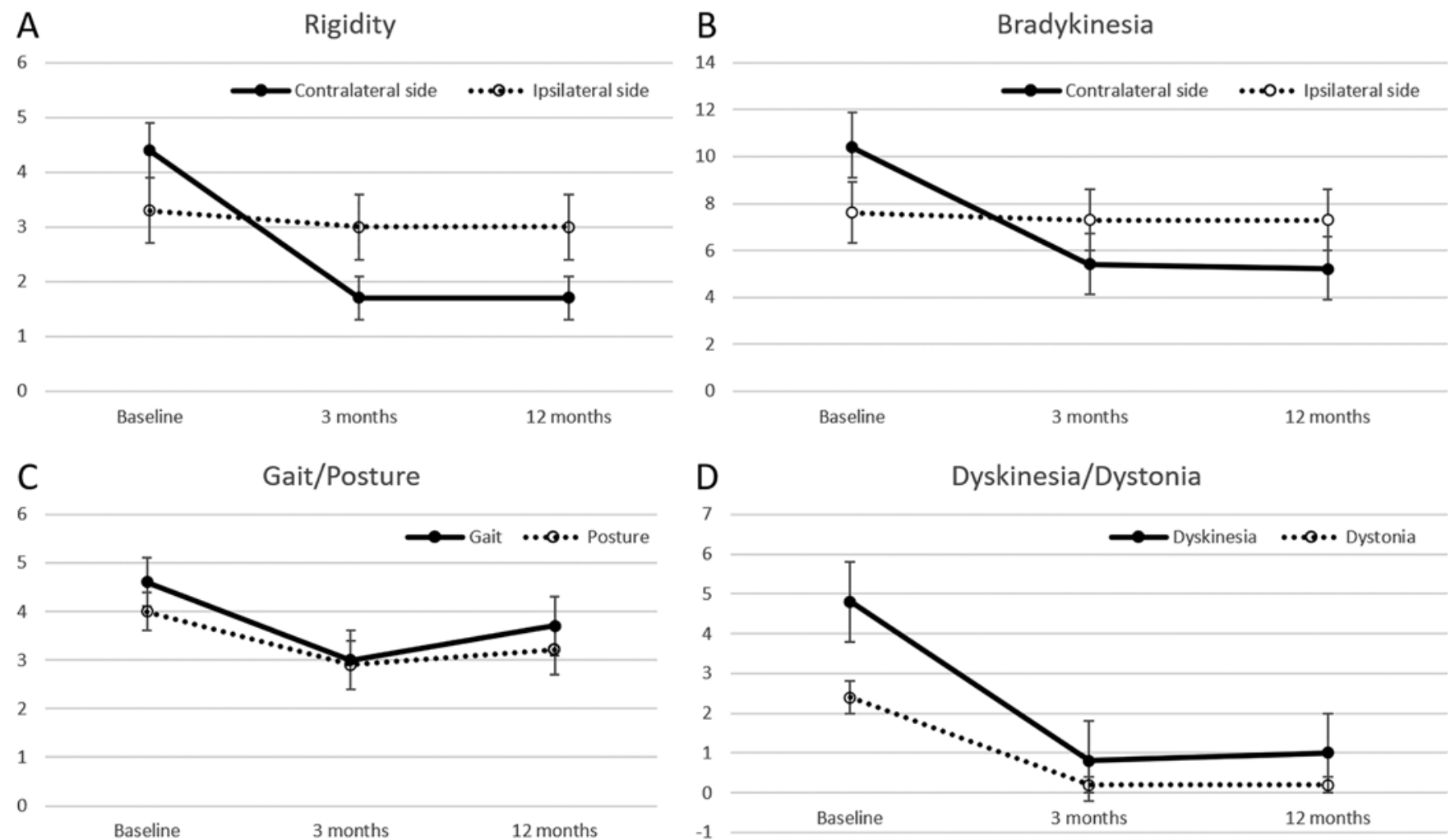

FIG. 2. Pre- and postoperative subscores of MDS-UPDRS Part III. Contralateral side rigidity (A) and bradykinesia (B), posture (C), dyskinesia (D), and dystonia (D) were significantly improved 12 months after surgery. Gait (C) also improved 12 months after surgery without statistical significance for the comparison of mean baseline and 12-month postoperative scores (Wilcoxon signedrank test). Error bars represent the standard error of the mean. 

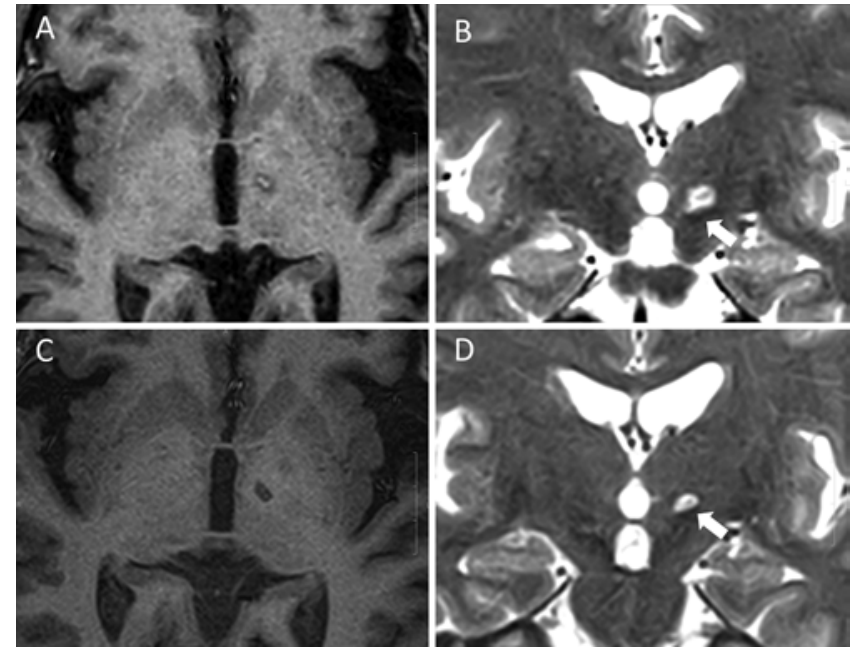

FIG. 3. Follow-up T1- and T2-weighted MR images at 0 (A and B) and 3 (C and D) months after PTT showing the lesion above the subthalamic nucleus (white arrows).

tients was unknown. The mean follow-up period was 2 months (range 1-3 months). The pre- and postoperative total MDS-UPDRS Part III scores at each last available evaluation were $35 \pm 3.4$ and $26.5 \pm 3.3$, respectively (24.3\% improvement). No significant postoperative complications were observed in these patients.

\section{Discussion}

In this study, we sought to evaluate prospectively the efficacy and safety of unilateral PTT for AR-PD. One year after surgery, patients showed improvements in the clinician-scored motor evaluation, contralateral side rigidity and bradykinesia (50\% improvement), dyskinesia (79.2\% improvement), and dystonia (91.7\% improvement). While posture significantly improved with a $20 \%$ reduction in scores ( $p=0.038$ ), no significant improvement was found in gait ( $p=0.066)$. The LEDD was not changed. In terms of the procedure's safety, 1 patient had a chronic subdural hematoma 2 months after the surgery. No other serious adverse events were reported.

Two recent studies using radiofrequency PTT/campotomy for PD have reported superior results in treating the cardinal motor symptoms of PD including rigidity, tremor, bradykinesia, gait, and posture. ${ }^{12,15}$ Aufenberg et al. reported the effects of radiofrequency PTT in 22 unilateral and 19 bilateral cases, observing an $87 \%$ improvement in tremor, an $82.4 \%$ improvement in rigidity, 58\% improvement in bradykinesia, $88 \%$ improvement in dyskinesia, and $33 \%$ improvement in axial symptoms at 22 months' follow-up. ${ }^{12}$ Additionally, they reported a $68 \%$ reduction in the LEDD. Gallay et al. ${ }^{6}$ reported unilateral/bilateral PTT using focused ultrasound for 51 patients with PD. The results were similar to those of Aufenberg et al., ${ }^{12}$ showing an $84 \%$ improvement in tremor, $70 \%$ in rigidity, $73 \%$ in distal limb bradykinesia, and $55 \%$ reduction of levodopa intake. Similarly, Godinho et al. ${ }^{15}$ reported a detailed analysis of the effects of unilateral campotomy on motor disturbances, as well as neuropsychological func-
TABLE 3. Adverse events

\begin{tabular}{lcc}
\hline \multirow{2}{*}{ Event } & \multicolumn{2}{c}{ No. of Patients } \\
\cline { 2 - 3 } & Temporary & Persistent \\
\hline Somnolence & 6 & \\
\hline Hypophonia & & \\
\hline Drooling & 1 & 1 \\
\hline Eyelid apraxia & 1 & 3 \\
\hline Fatigue & & \\
\hline Decreased response to levodopa & & \\
\hline Chronic subdural hemorrhage & 1 & \\
\hline
\end{tabular}

tions and quality of life, in a well-designed prospective study. In their report, a $43.9 \%$ improvement was observed in the clinician-scored motor evaluation, a $65.7 \%$ improvement in tremor, an $87.8 \%$ improvement in rigidity, a $68 \%$ improvement in bradykinesia, an $82.4 \%$ improvement in dyskinesia, and a $31.9 \%$ improvement in gait in 12 cases at the 2-year follow-up. Additionally, the patients' quality of life was also improved by $37.8 \%$. The previous studies reported a greater degree of improvement in the clinicianscored motor evaluation (MDS-UPDRS Part III score) than we did in the current study. ${ }^{6,12,15}$

A possible reason for this difference in motor outcomes may be patient selection. PD has three subtypes: tremor dominant (TD), AR, and mixed (MX) ${ }^{16}$ Several studies have demonstrated morphological and physiological differences between AR-PD and TD-PD ${ }^{17-20}$ Rapid disease progression, poorer response to levodopa, and the likelihood of dementia are greater in AR-PD than in TD-PD and MX-PD. ${ }^{16,21}$ Loss of dopaminergic neurons in the caudal GPi in AR-PD is more pronounced and widespread than in TD-PD and MX-PD. ${ }^{22}$ AR-PD or postural instability and gait difficulty subtypes have been reported to be less likely to respond to GPi-DBS or STN-DBS compared to TD-PD. ${ }^{23,24}$ In Godinho and colleagues' study, ${ }^{15} 8$ patients (66.7\%) had TD-PD and 4 had AR-PD. In Aufenberg and associates' study, ${ }^{12}$ no patient had pure AR-PD; all the evaluated patients had significant resting tremor. In Gallay and colleagues' study, 5 patients (10\%) had AR-PD, 9 (18\%) had TD-PD, and 37 (72\%) had MX-PD. ${ }^{6}$ Conversely, all patients in the present study had AR-PD without significant resting tremor. This suggests that the response to surgery may be different among AR-, TD-, and MX-PD patients.

A significant reduction in the LEDD is an important and valuable issue for patients and is the reason for the preference for STN-DBS over GPi-DBS. Our study showed no significant reduction in LEDD after unilateral PTT, which is concordant with findings in Godinho and colleagues' study, ${ }_{15}$ which reported a 9.3\% reduction in LEDD. In our study, 3 patients had a reduction in LEDD at 3 months, and 2 of the 3 returned to their preoperative LEDD by 1 year after surgery. Another 3 patients exhibited a reduced response to levodopa, including a shorter levodopa effect duration and a longer time to become effective, which resulted in an increased LEDD to maintain their preoperative daily living activities. Two of these patients required 
their dose frequency to be increased from 3 to 4 times per day. The preoperative and 3-month postoperative LEDDs in the 3 patients were 900,750 , and $450 \mathrm{mg}$; and 1200 , 900 , and $500 \mathrm{mg}$, respectively. The LEDD was unchanged at the 1-year evaluation. The etiology of this reduced response to levodopa after surgery is not known. However, a similar phenomenon was reported by Intemann et al., ${ }^{25}$ suggesting that a gradual reduction in responsiveness to levodopa may be a side effect of pallidotomy. In particular, bilateral interventions may be associated with a significant prolonged reduction in LEDD. Only Jeanmonod's groups (both the Aufenberg et al..$^{12}$ and the Gallay et al. ${ }^{6}$ studies) have performed bilateral PTT by radiofrequency and focused ultrasound ablation. However, they did not report on the differences in clinical results between unilateral and bilateral PTT. Aufenberg et al. ${ }^{12}$ reported a $68 \%$ decrease in LEDD at an average of 22 months' postoperative evaluation after radiofrequency PTT, including bilateral procedures. Gallay et al. ${ }^{6}$ reported a $55 \%$ reduction in LEDD at the 1-year postoperative evaluation after focused ultrasound PTT, including bilateral procedures. These results are markedly different from those of both the Godinho et al. study ${ }^{15}$ and our present study, both of which included only unilateral procedures.

Among the various complications noted in the present study, we consider the decreased response to levodopa to be the most serious adverse effect, and this has not been reported by previous studies using PTT. Somnolence was noted in $60 \%$ of patients, consistent with previous reports. ${ }^{6,15}$ Apraxia of lid opening was noted in 1 patient. While the present study supports the safety of unilateral PTT/campotomy, the safety of bilateral PTT/campotomy remains unknown. In our previous study of unilateral PTT combined with contralateral pallidotomy for dystonia, we reported that more than half $(54.5 \%)$ of the patients experienced significant prolonged hypophonia. ${ }^{26}$ In the present study, only 2 patients (20\%) reported significant hypophonia, which persisted at the 1-year evaluation. However, the degree of hypophonia was very mild and did not interfere with their daily communication. Patients with PD are likely to have speech and swallowing problems including hypophonia, which suggests that they are vulnerable to surgical complications following bilateral procedures. Similarly, Gallay et al. ${ }^{6}$ reported a significant worse rate of hypophonia after bilateral PTT using focused ultrasound. Therefore, we would not perform bilateral PTT for PD. The effects of unilateral PTT are expected to decrease gradually with time. For those patients who require additional surgery to provide further improvement, DBS may be better to ensure both safety and efficacy. We did not evaluate psychiatric and neuropsychological conditions after surgery. While Godinho et al. have previously reported that these conditions did not significantly deteriorate, Gallay et al. reported a transient anxio-depressive state in 1 patient (2\%). ${ }^{6,15}$ Further, Aufenberg et al. reported that anxio-depressive and reduced activity states were observed in $6(14.6 \%)$ and $4(9.8 \%)$ patients, respectively. ${ }^{12} \mathrm{~A}$ larger number of patients is needed to elucidate the effects of PTT on patients' psychiatric and psychological states.

This study has some limitations. The open-label trial design may have influenced reporting and the measure- ment of outcomes and introduced a bias in the results. In addition, the limited sample size reduced the power of the study to detect small to moderate treatment effects. Tractography can improve stereotactic targeting; however, we were unable to visualize the course of the pallidothalamic tract using tractography. Finally, the 1-year follow-up is relatively short for $\mathrm{PD}$, which is a slowly progressive disease.

\section{Conclusions}

This study revealed improved contralateral side rigidity and bradykinesia, and dyskinesia following unilateral PTT for patients with AR-PD. No serious adverse effects were associated with the procedure. In the future, a larger sample of patients together with a longer follow-up and blinded assessments is needed to elaborate more precisely the safety and efficacy of PTT for AR-PD.

\section{References}

1. Follett KA, Weaver FM, Stern M, et al. Pallidal versus subthalamic deep-brain stimulation for Parkinson's disease. $N$ Engl J Med. 2010;362(22):2077-2091.

2. Deuschl G, Schade-Brittinger C, Krack P, et al. A randomized trial of deep-brain stimulation for Parkinson's disease. $N$ Engl J Med. 2006;355(9):896-908.

3. Obeso JA, Olanow CW, Rodriguez-Oroz MC, et al. Deepbrain stimulation of the subthalamic nucleus or the pars interna of the globus pallidus in Parkinson's disease. $N$ Engl J Med. 2001;345(13):956-963.

4. Lozano AM, Lang AE, Galvez-Jimenez N, et al. Effect of GPi pallidotomy on motor function in Parkinson's disease. Lancet. 1995;346(8987):1383-1387.

5. Laitinen LV, Bergenheim AT, Hariz MI. Leksell's posteroventral pallidotomy in the treatment of Parkinson's disease. $J$ Neurosurg. 1992;76(1):53-61.

6. Gallay MN, Moser D, Rossi F, et al. MRgFUS pallidothalamic tractotomy for chronic therapy-resistant Parkinson's disease in 51 consecutive patients: single center experience. Front Surg. 2020;6:76.

7. Jung NY, Park CK, Kim M, et al. The efficacy and limits of magnetic resonance-guided focused ultrasound pallidotomy for Parkinson's disease: a Phase I clinical trial. J Neurosurg. 2019;130(6):1853-1861.

8. Martínez-Fernández R, Rodríguez-Rojas R, Del Álamo M, et al. Focused ultrasound subthalamotomy in patients with asymmetric Parkinson's disease: a pilot study. Lancet Neurol. 2018;17(1):54-63.

9. Chung BS, Park JS. Whole course of pallidothalamic tracts identified on the sectioned images and surface models. Clin Anat. 2020;33(1):66-76.

10. Gallay MN, Jeanmonod D, Liu J, Morel A. Human pallidothalamic and cerebellothalamic tracts: anatomical basis for functional stereotactic neurosurgery. Brain Struct Funct. 2008;212(6):443-463.

11. Neudorfer C, Maarouf M. Neuroanatomical background and functional considerations for stereotactic interventions in the H fields of Forel. Brain Struct Funct. 2018;223(1):17-30.

12. Aufenberg C, Sarnthein J, Morel A, et al. A revival of Spiegel's campotomy: long term results of the stereotactic pallidothalamic tractotomy against the parkinsonian thalamocortical dysrhythmia. Thalamus Relat Syst. 2005;3(2):121-132.

13. Spiegel EA, Wycis HT, Szekely EG, et al. Campotomy in various extrapyramidal disorders. J Neurosurg. 1963;20: 871-884.

14. Magara A, Bühler R, Moser D, et al. First experience with 
MR-guided focused ultrasound in the treatment of Parkinson's disease. J Ther Ultrasound. 2014;2:11.

15. Godinho F, Magnin M, Filho PT, et al. Stereotactic lesion in the Forel's field H: a 2-years prospective open-label study on motor and nonmotor symptoms, neuropsychological functions, and quality of life in Parkinson disease. Neurosurgery. 2019;85(4):E650-E659.

16. Rajput AH, Voll A, Rajput ML, et al. Course in Parkinson disease subtypes: a 39-year clinicopathologic study. Neurology. 2009;73(3):206-212.

17. Zhang J, Wei L, Hu X, et al. Akinetic-rigid and tremordominant Parkinson's disease patients show different patterns of intrinsic brain activity. Parkinsonism Relat Disord. 2015; 21(1):23-30.

18. Schillaci O, Chiaravalloti A, Pierantozzi M, et al. Different patterns of nigrostriatal degeneration in tremor type versus the akinetic-rigid and mixed types of Parkinson's disease at the early stages: molecular imaging with 123I-FP-CIT SPECT. Int J Mol Med. 2011;28(5):881-886.

19. Kaasinen V, Kinos M, Joutsa J, et al. Differences in striatal dopamine transporter density between tremor dominant and non-tremor Parkinson's disease. Eur J Nucl Med Mol Imaging. 2014;41(10):1931-1937.

20. Benninger DH, Thees S, Kollias SS, et al. Morphological differences in Parkinson's disease with and without rest tremor. J Neurol. 2009;256(2):256-263.

21. Rajput AH, Rajput ML, Ferguson LW, Rajput A. Baseline motor findings and Parkinson disease prognostic subtypes. Neurology. 2017;89(2):138-143.

22. Rajput AH, Sitte HH, Rajput A, et al. Globus pallidus dopamine and Parkinson motor subtypes: clinical and brain biochemical correlation. Neurology. 2008;70(16 Pt 2):1403-1410.

23. Tsuboi T, Lemos Melo Lobo Jofili Lopes J, Patel B, et al. Parkinson's disease motor subtypes and bilateral GPi deep brain stimulation: one-year outcomes. Parkinsonism Relat Disord. 2020;75:7-13.

24. Katz M, Luciano MS, Carlson K, et al. Differential effects of deep brain stimulation target on motor subtypes in Parkinson's disease. Ann Neurol. 2015;77(4):710-719.
25. Intemann PM, Masterman D, Subramanian I, et al. Staged bilateral pallidotomy for treatment of Parkinson disease. $J$ Neurosurg. 2001;94(3):437-444.

26. Horisawa S, Fukui A, Tanaka Y, et al. Pallidothalamic tractotomy (Forel's field H1-tomy) for dystonia: preliminary results. World Neurosurg. 2019;129:e851-e856.

\section{Disclosures}

The authors report no conflict of interest concerning the materials or methods used in this study or the findings specified in this paper.

\section{Author Contributions}

Conception and design: Horisawa, Iijima, Kawamata, Taira. Acquisition of data: Horisawa, Fukui, Yamahata, Tanaka, Kuwano, Momosaki, Iijima, Nanke, Taira. Analysis and interpretation of data: Horisawa, Taira. Drafting the article: Horisawa. Critically revising the article: Horisawa. Reviewed submitted version of manuscript: Horisawa. Approved the final version of the manuscript on behalf of all authors: Horisawa. Statistical analysis: Horisawa, Fukui. Administrative/technical/ material support: Iijima, Taira. Study supervision: Iijima, Kawamata, Taira.

\section{Supplemental Information \\ Videos}

Video 1. https://vimeo.com/453250738.

Video 2. https://vimeo.com/453250752.

Video 3. https://vimeo.com/453250758.

\section{Correspondence}

Shiro Horisawa: Neurological Institute, Tokyo Women's Medical University, Tokyo, Japan. neurosurgery21@yahoo.co.jp. 